\title{
Dynamic Trust Relationship Quantization Model Based on Multiple Attribute Decision
}

\author{
Minsheng Tan ${ }^{*}$, Miao Guo, Ting Xiang, Hui Zhao and Chencheng Wang \\ School of Computer Science and Technology, University of South China, Hengyang, Hunan, China \\ ${ }^{*}$ Corresponding author
}

\begin{abstract}
Dynamic trust relationship making quantization model based on multiple attribute decision has been researched in this paper. Considering the problem that all information from nodes feedback would be not true or reliable, this paper improves a new rule to calculate the feedback trust and proposes a new quantization model on dynamic trust-relationship based on improved feedback trust. On this basis, from the complex structure characteristics of network, this paper introduces the nodes similarity and proposes a improved model of dynamic trust-relationship based on multi-attribution. The simulation experiment showed that the new model have better dynamic adaptability and get a better accuracy and stability of trust evaluation of network.
\end{abstract}

Keywords-trusted network; trust-relationship; multi-attribution; quantization; node similarity

\section{INTRODUCTION}

The development of network brings a lot of convenience to people's life, however, there exists a lot of potential safety hazards, such as information intercept, mail attack, Internet fraud and so on, and the problem of network security also cause some security threats on the state of the economy, society, humanity etc. Establish a secure service system with the case of the network inherent vulnerability, human error factors, network attack and destruction, it still be able to do a variety of service performance which can be predicted, security service can be survived, behavioral results can be controlled, it means that to build a trusted network, has gradually become a research hotspot. ${ }^{[1][2][3]}$

The article takes the credibility of the feedback information with the unsafe factors of the network into consideration to improve the quantification accuracy of dynamic trust relationship. Based on the characteristics of network structure on network performance, node similarity is introduced to improve the accuracy and stability of the quantitative model of dynamic trust relationships.

\section{RELATED RESEARCH}

So far, there has been such a lot of research network for dynamic trust relationship quantitative model in the country. Li Xiaoyong ${ }^{[4]}$, who put forward the dynamic relationship of trust based on multi-attribute model. The model thought properties may impact the entire network, such as Direct Trust, the History of Trust, Risk Function, Feedback Trust and the complexity and uncertainty of the network trust relationships. Li Jingtao ${ }^{[5]}$ proposed a global trust model (SWRTrust) based on the peer-to-peer environment of the similarity weighting recommendation of the behavior of node score. The model illustrated the view of the consistency of these nodes to other network by proposing the score of the node behavior similar. Yan Ying ${ }^{[6]}$ proposed a global trust model (GSTrust) based on similarity weighted recommended.

Most of the existing trust relationship of quantitative models evaluate the whole network by quantifying the behavior trust among nodes, without considering the influences of networks' structure characteristics on the trust evaluation of the network relationship and the role the trust value of key nodes have played in the whole network. There exist some defects, such as the network trust evaluation is not comprehensive, the scope of application is limited, the node trust value is not stable and the realization mechanism is complex and so on.

\section{Quantitative Model Based on Multi-Attribute DECISION-MAKING DYNAMIC TRUST RELATIONSHIPS}

\section{A. Model Construction}

Definition $1 \mathrm{~N}$ bodies in network are denoted by $\mathrm{x}_{\mathrm{i}}(\mathrm{i}=1,2$, $3 \ldots n$ ), the trust relationships by $x_{i}$ to $x_{j}$ in the network are defined as a 9-element data structures:

$$
\operatorname{trust}\left(x_{i}, x_{j}\right)=\left[\Gamma, D, I, R, H, S, S^{\prime}, C, t\right]
$$

In(1), T means the overall trust in nodes; D means direct trust value of the bodies ; I means feedback trust of the other bodies $; \mathrm{R}$ means the risk function under the corresponding service ;H means historical trust in the interactive behavior ; $\mathrm{S}$ is a service provided by SP ;According to the mapping relationship of the quality of service $S$ and trust degree $I$, the service level of SR may be obtained is determined by the $\Gamma$ 's value; $S$ ' means the similarity in nodes based on resource allocation; $C$ means the context of the node interaction, it makes sense only in the specific context and by the general network interaction behavior, and it isn't defined by special means; $t$ means interactive time stamp.

Model evaluation framework in Figure I. Record the direct trust value of nodes of the interactive behavior after the interactive behavior has acted in the network nodes, then combine with the feedback trust of other nodes, the interaction value at risk as well as its prior interactive 
historical trust value, considering the node similarity under the network structure, then get the new trust value according to certain rules for polymerization, and updating the trust knowledge base in order provide effective evidence for the new record and the update of the interactive behavior.

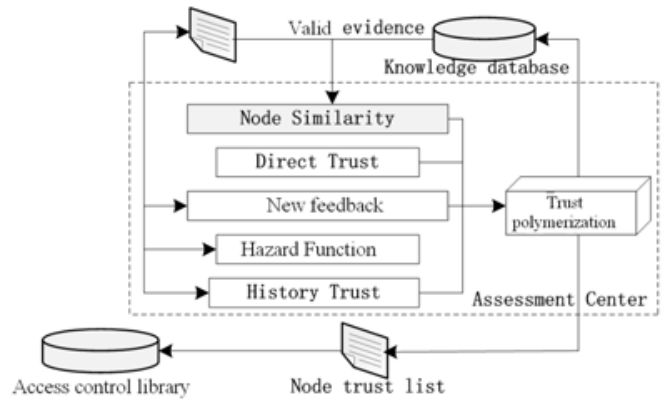

FIGURE I. MODEL EVALUATION FRAMEWORK

Except the above-described various attributes, the bodies in the network contain a mapping relationship between the service and the level of trust. Assume that there are passessment grades such as $\mathrm{c}_{1}, \mathrm{c}_{2,1 \ldots \ldots} \mathrm{s}_{\mathrm{p}}$, in the overall trust $T^{\prime}\left(x_{1}, z_{1}, x_{n}\right)$, and in them, $0 \leq c_{p} \leq 1 \quad(p=1,2, \ldots p)$. The simulation is adopted by $\mathrm{U}=\{0,0.2,0.4, \mathrm{O} .6,0.8,1\}$, the appropriate trust level mapping shown in Table I.

TABLE I. THE MAIN TRUST LEVEL MAPPING

\begin{tabular}{|c|c|c|}
\hline ID & The Trust Value & The Trust Relationship \\
\hline 1 & {$[0,0.2]$} & Very Low \\
\hline 2 & {$[0.2,0.4)$} & Low \\
\hline 3 & {$[0.4,0.6)$} & Normal \\
\hline 4 & {$[0.6,0.8)$} & High \\
\hline 5 & {$[0.8,1.0)$} & Very High \\
\hline
\end{tabular}

Definition 2 Assume that bodies can provide $\mathrm{k}$ levels of service, such as $\mathrm{S}=\left\{\mathbf{5}_{1}, \mathbf{5}_{\mathbf{n}}, \ldots, \mathbf{5}_{k}\right\}$, and $\mathrm{S}$ is a class of ordered partition, the mapping function between the $\mathrm{S}$ and the overall trust $\Gamma$ expressed by $\psi$ as:

$$
\begin{aligned}
& \Psi \quad\left(\Gamma\left(\mathrm{x}_{i}, x_{j}, s, n\right)\right)=\left\{_{\mathrm{k}}, C_{p} \Gamma\left(x_{i}, x_{j}, s, n\right) \leq 1\right. \\
& s_{k-1}, C_{p-1} \leq \Gamma\left(x_{i}, x_{j}, s_{p}, n\right) \leq c_{p} \\
& \ldots \\
& s_{2}, c_{1} \leq \Gamma\left(x_{i}, x_{j}, s, n\right) \leq c_{2} \\
& s_{1}, 0 \leq \Gamma\left(x_{i}, x_{j}, s, n\right) \leq c_{1}
\end{aligned}
$$

\section{B. Quantification of Decision Attribute}

Definition 3 Direct trust The trust satisfaction evaluation $\mathrm{E}_{\mathrm{ij}}$ is produced by h-direct interactions with main body $\mathrm{x}_{\mathrm{i}}$ and

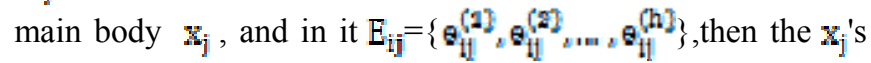
direct trust by xi as follows:

$$
\mathrm{D}\left(\mathrm{x}_{\mathrm{i},}, \mathrm{x}_{\mathrm{j}} \mathrm{n}\right)=\frac{\sum_{\mathrm{t}=\mathrm{k}}^{\mathrm{n}-1} \mathrm{~d}^{(\mathrm{t})}}{\mathrm{n}-\mathrm{k}-1}+\mathrm{d}^{(\text {new })}
$$

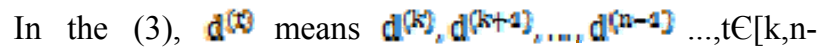
1] ,they are the trust behavior evaluation based on the nearest consecutive $n-k-1$ timestamps by subject $x_{i}$ to main body $\mathrm{x}_{\mathrm{j}} \cdot \mathrm{d}^{\text {(new }} \mathrm{m}$ means the trust value-added after the interactive behavior, and if two subjects never happened interactive behavior, or at the beginning of the direct trust value with a fixed time interval of the dynamic assessment, the value of $\mathrm{d}($ new $)$ is 0 .when $\mathrm{k} \in[1, \mathrm{n}-1]$, the greater its value is, means the smaller the assessment impact of the current direct trust about the prior interactive behavior which occurred; when $\mathrm{k}=\mathrm{n}-1$, the previous record would be completely forgotten, and will not impact on the trust evaluation of the current behavior ; when $\mathrm{k}=1$, it means that the previous interactive behavior were recorded and maximize impacted the current direct trust degree evaluation.

Definition 4 Historical Function Historical interaction between the two bodies in the network, there is such a function:

$$
H\left(X_{i}, X_{j}\right)=\max \left\{\frac{w_{1}}{w_{1}+w_{2}} \operatorname{Succ}\left(X_{i}, X_{j}\right)-\frac{w_{2}}{w_{1}, w_{2}} \operatorname{Fail}\left(X_{i}, X_{j}\right), 0\right\}
$$

In (4), Succ $\left(\mathrm{x}_{1}, \mathrm{x}_{\mathrm{j}}\right)$ and Fail $\left(\mathrm{x}_{1,}, \mathrm{x}_{\mathrm{j}}\right)$ express, respectively, the number of success and failure of the interaction between main body $X_{i}$ and main body $x_{j}$ in some timestamps recorded by the system recently. The simulation introduced $\omega_{1}$ and $\omega_{2}$ as penalty factors, according their sizes to reflect the punitive of interactive behavior among the bodies in the model. When $\omega_{1}>\omega_{z}$, the model reflects the incentive of the successful interactive behavior; when $\mathbb{w}_{1}<w_{2}$, the model reflects the punitive of the failure interactive behavior; the trust value is increased slowly and decreased rapidly, the greater of the ratio with $\boldsymbol{\sigma}_{2}$ and $\boldsymbol{\omega}_{1}$, the more severe of the system punitive measures, it can effectively avoid that some malicious bodies attack on the system and some of the bodies free to leave the system or change the quality of service randomly, resulting the transaction fails.

From an economic perspective, there is a certain risk value in every interactive behavior.

Definition 5 Hazard Function From a point of service, scholars use the venture capital principle of the economics, define the Hazard function in the network as follows:

$$
\begin{aligned}
& \mathrm{R}\left(\mathrm{x}_{\mathrm{i}}, \mathrm{x}_{\mathrm{j}}\right)=1-\mathrm{s}_{\mathrm{j}}\left(1-\Gamma\left(\mathrm{x}_{\mathrm{i}}, \mathrm{x}_{\mathrm{j}}, \mathrm{s}, \mathrm{t}-1\right)\right) \\
= & 1-\psi\left(\Gamma\left(x_{i}, x_{j}, s, t-1\right)\right)\left[1-\Gamma\left(x_{i}, x_{j}, s, t-1\right)\right]
\end{aligned}
$$

According to the service and the corresponding level of trust, through (5) we can get the interaction value at risk. Among them, $R\left(x_{i}, x_{j}\right)$ indicates the risk value of the request 
service of the main body $X_{i}$ to $X_{j}$. According to economic investment risk principle and experience, the quality of the service requests higher grades, the interactive risk is bigger, so risk is proportional to $s_{j} \cdot \Gamma\left(x_{i}, x_{j}, s, t-1\right)$ shows that $x_{i}$ has a general trust value to $x_{j}$ on the recent time stamp ,trust degree of the main body is inversely proportional to the interactive risk, so $1-\Gamma\left(x_{i}, x_{j}, s, t-1\right)$ is proportional to the value of risk. $X_{i}$

Definition 6 Feedback trust reflects the level of service promised by the main body to other subject of the network and the trust degree of the other main body to the main body. Assume that the feedback collection is $\left\{w_{1}, w_{2}, \ldots, w_{L}\right\}$, $D\left(w_{k}, x_{j}\right)$ expresses the trust degree of the kth responder to $x_{j}$ in collection. Define feedback trust as:

$$
\mathrm{I}\left(x_{i}, x_{j}\right)=\frac{\sum_{k=1}^{L}\left(\rho\left(W_{k}\right) \times D\left(W_{k}, x_{j}\right)\right)}{\sum_{k=1}^{L} \rho\left(W_{k}\right)}
$$

In (6), $\mathrm{L}$ is the number of main body for feedback, $\rho\left(W_{k}\right)$ is the feedback weighted function, defined as follows:

$$
\rho\left(w_{k}\right)\left\{\begin{array}{c}
1, l=1 \\
\prod_{d=0}^{l} D\left(x_{d}, x_{\text {suc }}\right)
\end{array}\right.
$$

In (7), L shows the providers' class of feedback, $D\left(x_{d}, x_{\text {succ }}\right)$ indicates that the direct trust of main body $x_{d}$ to its successor body $x_{\text {succ }}$ on the trust way from main body $x_{i}$ to main body $x_{j}$. Considering the credibility of the feedback information, this paper proposes a new calculation method for the feedback trust. To L feedback subjects, the respondents $w_{k}$ of feedback information for the credibility of the record is $F\left(w_{k}\right)$, the respondents weighted function will redefine for $\rho^{\prime}\left(W_{k}\right)$ :

$$
\rho^{\prime}\left(W_{k}\right)=\rho\left(W_{k}\right) \times F\left(W_{k}\right)
$$

The definition of credibility $F\left(W_{k}\right)$ of the feedback information is:

$$
F\left(W_{k}\right)=\frac{T\left(W_{k}, x_{i}\right)}{\sum_{m=1}^{l} T\left(W_{m}, x_{i}\right)}
$$

Feedback information relate with not only network series with feedback nodes, but also the credibility of the feedback information, so the new trust calculation rules for feedback is:

$$
I\left(x_{i}, x_{j}\right)=\frac{\sum_{k=1}^{L}\left(\rho^{\prime}\left(W_{k}\right) \times D\left(W_{k}, x_{j}\right)\right)}{\sum_{k=1}^{L} \rho^{\prime}\left(W_{k}\right)}
$$

Definition 7 Node similarity Hypothetically every media has a unit of resources and it will be evenly distributed to its neighbors, then can accept the resources and the namber of the rescurces can be defined as the similarities of node $X_{i}$ and node $X_{j}$ :

$$
S_{x_{i} x_{j}}^{\prime}=\sum_{z \in \Phi\left(x_{i}\right) \cap \Phi\left(x_{j}\right)} \frac{1}{d(z)}
$$

In (11) $\Phi\left(x_{i}\right)$ shows the neighbor collection of $x_{i}$, $\Phi\left(x_{j}\right)$ means the neighbor collection of $x_{j}, z$ indicates the collection of the common neighbor of $x_{i}$ and $x_{j}, \mathrm{~d}(\mathrm{z})$ expresses its degree.

Definition 8 Overall degree of polymerization According to the different attribute definitions of above, in this paper the overall level of trust polymerization function is defined as:

$$
\Gamma\left(x_{i}, x_{j}, s, t\right)=\sum_{m=1}^{M} \varpi_{m} X_{m}\left(x_{i}, x_{j}\right)
$$

Among them $\mathrm{s}$ is the service quality level provided by $x_{i}, \mathrm{t}$ is the interactive time stamp, $X_{m}\left(x_{i}, x_{j}\right)$ is the decision attribute value in the formula of the front, $\varpi_{m}$ is the weight of evaluating each decision attribute in total trust .

\section{The Simulation RESUlts AND ANAlysis}

This paper use simulation platform Netlogo, which is particularly suitable for the complex system modeling simulation with the time evolution. Netlogo is a free software proposed by northwestern university links study with computer modeling research and development center, the simulations were completed by the programming in Java language and can be operated in different platforms. The 
model established in this paper references the original model as in [7].

According to the dynamic characteristic of network, simulation settings parameters are: service request frequency SRF means the node numbers that request service every moment of network. Service dynamic frequency SCF shows how many nodes switch between GS and BS in the network. Community dynamic frequency SDF expresses how many network nodes exist instability, how many can leave or join the network to the community at will. Related parameters and the simulation are designed as Table II and Table III shows.

TABLE II. SIMULATION PARAMETER

\begin{tabular}{|c|c|c|}
\hline parameter & value & describe \\
\hline Node of Net & 1000 & Network subject number \\
\hline $\mathrm{S}$ & 2000 & Operation test times \\
\hline $\mathrm{k}$ & 10 & Record interactive number \\
\hline$\varpi_{1}, \varpi_{2}$ & $0.2,0.8$ & Interactive punish factor \\
\hline 1 & 3 & Feedback subject series \\
\hline
\end{tabular}

The accuracy of improved dynamic trust quantization model is measured by the interactive success rate (SSP) of the network's main body. For a dynamic network system, a higher success rate of interaction also explains a better trust decision accuracy, meanwhile has a better dynamic adaptability.'

$$
\Omega(t)=\frac{A_{g}(t)}{B_{\text {total }}(t)}
$$

Definition $9 \Omega(t)$ expresses Interactive success rate and description in math as in (13)

Among them, $A_{g}(t)$ says the numbers of success of interaction occurred in the system recorded in there simulation experiment at the $\mathrm{t}$ moment, $B_{\text {total }}(t)$ indicates the total number of interaction. Figure 2 and Figure 3 separately described the results in stability and busy two states of the network. In the figure, Old Model represents the original multiple attributes dynamic trust quantization model, and IOTKDTQ represents the improved Model.

TABLE III. NETWORK NODE DESIGN

\begin{tabular}{|c|c|}
\hline role & type \\
\hline \multirow{3}{*}{$\begin{array}{c}\text { SP(Service } \\
\text { provider) }\end{array}$} & GS(Always provide reliable service) \\
\cline { 2 - 2 } & BS(Total denial of service) \\
\cline { 2 - 2 } FR(Responde & GS/BS \\
\cline { 2 - 2 } nt provider) & $\begin{array}{c}\text { E(According to the feedback expanding factor by } \\
\text { expanding the feedback information provided })\end{array}$ \\
\cline { 2 - 2 } & $\begin{array}{c}\mathrm{C} \text { (On gang nodes in the feedback information of } 1,0 \\
\text { to other node feedback) }\end{array}$ \\
\hline
\end{tabular}

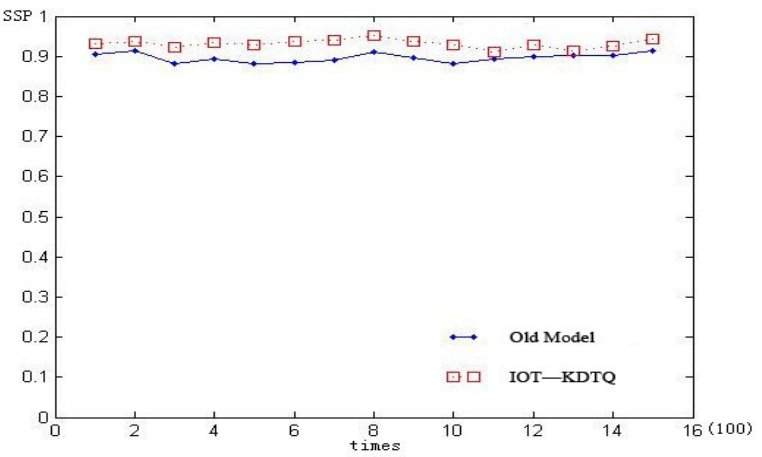

FIGURE II. NETWORK UNDER STEADY STATE

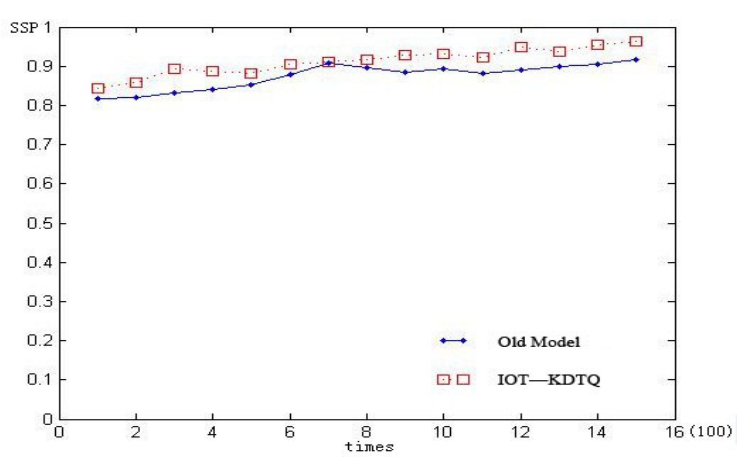

FIGURE III. NETWORK UNDER A BUSY STATE

As shown in Figure II, the comparison between improved multi-attribute decision-making model and the original multiple attribute decision model of interaction success rate in the stable state of network. The improved Model improves the stability of the network behavior, and interactive success rate also improved. In general, Model-3 interaction success rate is $90 \%$ or more, it also further verified node similarity on assessment of the influence of the network behavior. In Figure III, it compares the interaction of success between the improved model and the original multiple attribute decision model in the busy network state. It can be seen in the busy network state, the improved model considers the network structure characteristic similarity on the performance of the network nodes, it is more accurate positioning analysis $t$ feedback trust in evaluation of trust than the former model of interaction success rate increases about $5 \%$ or so, also more stable.

\section{CONCLUSION}

To solve the problem of insufficiency of the multiple attribute decision attribute research dynamic trust quantization model, the article proposed improved feedback trust. Considering the influence of structural characteristics of complex network on the performance of the network, the article introduced node similarity which combined with direct trust, historical trust, risk function and improved feedback trust. Also, this article take into account the trust relationship among subjects of quantitative network in many aspects, which provided a new theory basis for people to study the relationship of online trust quantification model. 


\section{ACKNOWLEDGMENT}

This work was supported by Project 61300234 of the National Science Foundation of China, the construct program of the key discipline in University of South China, the construct program of the key laboratory in University of South China, Project 2012KG71 of the Hengyang City Science and Technology Plan, key Project 14A121 of the Hunan Provincial Education Office Science Research of China.

\section{REFERENCES}

[1] Hujianli, Wuquanyuan, Zhoubin, Liujiahong, "A distributed based on feedback credibility P2P trust model", Journal of software, vol. 20, pp. 2885-2898, 2009.

[2] Sun Y, Yu W, Han Z, Liu KJR, "Trust modeling and evaluation in ad hoc networks", In: Proc.of the Global Telecommunications Conf Globecom 2005. Washington: IEEE Computer Society Press, pp. 1-10, in press.

[3] Josang A, Haller j, "Dirichlet Reputation Systems", In: Werner B eds. Proceedings of 2nd International Conference on Availability,Reliability and Security Vienna. Los Vaqueros: IEEE Computer Society, pp. 112119,2007 , in press.

[4] Lixiaoyong, Zhangshaogang, "Multiple attribute dynamic trust quantization model", Computer application, vol. 28, pp. 884-887, 2008.

[5] Lijingtao, Jingyinan, Xiaoxiaochun, Wangxueping, Zhanggendu, "Based on the similarity of the weighted recommended P2P environment of trust model", Journal of software, vol. 18, pp. 157-167, 2007.

[6] Yanying, Lirenfa, "P2P environment a credible access control model", Computer application, vol. 28, pp. 3194-3196, 2008.

[7] Liang zhen-Qiang, Shi Wei-Song, "Analysia of recommendations on trust inference in open environment", Journal of Performance Evaluation, vol. 65 , pp. 99-128, 2007. 